\title{
Targeted therapies for resectable lung adenocarcinoma: ADAURA opens for thoracic oncologic surgeons
}

\author{
David R. Jones, MD, ${ }^{\mathrm{a}}$ Yi-Long Wu, MD, ${ }^{\mathrm{b}}$ Masahiro Tsuboi, $\mathrm{MD},{ }^{\mathrm{c}}$ and Roy S. Herbst, MD, $\mathrm{PhD}^{\mathrm{d}}$
}

Feature Editor Note-The randomized ADAURA trial has shown a significant benefit to receiving third-generation EGFR-tyrosine kinase inhibitor, osimertinib, after resection of lung cancer with EGFR mutation. The $83 \%$ risk reduction in disease recurrence or death reported by the ADAURA trial is practice-changing, as never before has such a magnitude of benefit been reported in any previous adjuvant trial. Although overall survival has yet to be reported, the profound results seen in disease-free survival is likely a signal that a benefit will also be found in overall survival, we eagerly await maturation of these data for confirmation. With the publication of the ADAURA trial, the role of the thoracic surgeon becomes even more important, beyond the surgical care we deliver, as we must now ensure genetic profiling for resected lung cancer, ensure delivery of the appropriate adjuvant therapy (or therapies) if indicated, and we must ensure patient accrual to important future adjuvant and neoadjuvant trials studying such targeted therapies for there is great potential for tremendous benefit as seen in the ADAURA trial. To this end, knowledge of not only the ADAURA trial results but also of its implications becomes crucial. Thus, this expert opinion presented is of timely importance. Dr Jones, who needs no introduction, has assembled an all-star team of authors, including investigators from the ADAURA trial, Drs Wu, Tsuboi, and Herbst, to provide us with important insights regarding this high-impact publication. This is a must-read article, not only for thoracic surgeons but for any medical professional who cares for patients with lung cancer.

\section{Thomas Ng, MD}

The increasing use of next-generation sequencing to better understand oncogenic drivers in lung cancer has revolutionized the care of patients with locoregionally advanced and metastatic disease. In lung adenocarcinoma (LUAD), these include alterations in $K R A S, M E T, B R A F, R O S 1$, and fusions

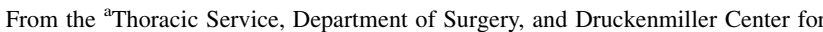
Lung Cancer Research, Memorial Sloan Kettering Cancer Center, New York, NY; ${ }^{b}$ Guangdong Lung Cancer Institute, Guangdong Provincial People's Hospital, Guangdong, China; 'National Cancer Center Hospital East, Kashiwa, Chiba, Japan; and ${ }^{\mathrm{d}}$ Yale School of Medicine and Yale Cancer Center, New Haven, Conn. Dr Jones is supported, in part, by National Cancer Institute P30 CA008748.

Received for publication Jan 25, 2021; revisions received Jan 29, 2021; accepted for publication Feb 2, 2021; available ahead of print Feb 9, 2021.

Address for reprints: David R. Jones, MD, Thoracic Service, Memorial Sloan Kettering Cancer Center, 1275 York Ave, New York, NY 10065 (E-mail: jonesd2@ mskcc.org).

J Thorac Cardiovasc Surg 2021;162:288-92

$0022-5223 / \$ 36.00$

Copyright (c) 2021 by The American Association for Thoracic Surgery

https://doi.org/10.1016/j.jtcvs.2021.02.008

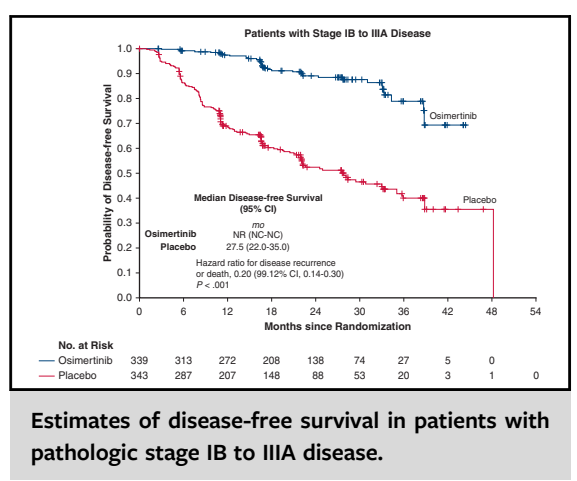

CENTRAL MESSAGE

The ADAURA trial of adjuvant

osimertinib in completely re-

sected pstage IB-IIIA, EGFR-

mutant lung adenocarcinoma is

the first "positive" adjuvant trial

since doublet chemotherapy

15 years ago.

This Invited Expert Opinion provides a perspec tive on the following paper: Source article: $N$ Engl J Med. 2020 Oct 29;383(18):1711-1723. doi.org/10.1056/NEJMoa2027071. Epub 2020 Sep 19.

See Commentaries on pages 293 and 294 .

in NTRK, ALK, and RET. However, the most well-studied remain the oncogenic activating mutations found in the EGFR gene on chromosome 7. The majority of targeted therapies for genomic perturbations identified in LUAD have focused on the inhibition of selected tyrosine kinases, which are corrupted in cancer and drive cell growth, proliferation, and cell survival. Specifically, for EGFR-driven tumors, exon 19 deletions and the exon 21 L858R point mutation are most predictive of response to tyrosine kinase inhibitor (TKI) therapies. Clinical use of TKIs for these EGFR mutations began with Food and Drug Administration approval of gefitinib in 2003 as second-line therapy for metastatic disease. In 2018, osimertinib, a third-generation TKI that selectively inhibits both EGFR-TKI-sensitizing and EGFR p.Thr790Met-resistance mutations and has excellent central nervous system (CNS) penetration, was approved as firstline therapy for stage IV disease. 
Complete surgical resection is the standard of care for patients with stage I-IIIA LUAD. However, even in patients with pathologic node-negative disease, distant recurrence of disease remains all too frequent, occurring in $11 \%$ of patients with pT $1 \mathrm{~b}$ tumors and $47 \%$ of patients with pT $2 \mathrm{~b} / 3$ LUAD. ${ }^{2}$ Recurrence rates are even greater for pathologic node-positive disease, particularly $\mathrm{pN} 2$ disease. To address this problem, multiple international randomized phase III clinical trials were performed in the late 1990s to examine adjuvant cisplatin-based doublet chemotherapy after complete resection. The pooled analysis of these studies by the Lung Adjuvant Cisplatin Evaluation demonstrated improved overall survival (OS) with adjuvant chemotherapy, particularly for pathologic stage II or III and node-positive disease. ${ }^{3}$ The overall hazard ratio (HR) of death was 0.89 (95\% confidence interval [CI], 0.82-0.96; $P=.005$ ), which corresponded to a 5 -year absolute benefit of $5.4 \%$ from adjuvant chemotherapy. ${ }^{3}$ Despite this relatively modest benefit, adjuvant cisplatin-based chemotherapy has been the standard of care in this population for the past 15 years.

\section{ADJUVANT EGFR-TARGETED THERAPY \\ CLINICAL TRIALS}

Given the near-universal testing for selected tumor mutations, such as EGFR, in metastatic LUAD and the development of newer TKI therapies with superior patient outcomes (with or without chemotherapy), it was logical to examine whether the benefits observed in stage IV disease could be translated to adjuvant-therapy care paradigms in patients with $E G F R$-mutant LUAD with surgically resectable disease. The first robust study to examine adjuvant TKI therapy was the phase III randomized RADIANT trial, which compared adjuvant erlotinib for 2 years with placebo in pathologic stage IB-IIIA LUAD, with disease-free survival (DFS) the primary end point. ${ }^{4}$ Eighty percent of accrued patients were white; $17 \%$ were of Asian descent. Patients were not stratified by EGFR mutational status-only EGFR immunohistochemistry and mRNA levels by fluorescence in situ hybridization analysis. Unsurprisingly, no benefit for adjuvant erlotinib after complete surgical resection was observed.

EVAN was a small phase II trial from China that investigated whether adjuvant erlotinib improves 2-year DFS, compared with doublet-based chemotherapy, in EGFR mutation-positive stage IIIA non-small cell lung cancer (NSCLC). ${ }^{5}$ Two-year DFS was $81 \%(95 \%$ CI, 69.6\%$93.1 \%)$ in the erlotinib group versus $45 \%(95 \% \mathrm{CI}$, $26.9 \%-62.4 \%$ ) in the chemotherapy group (relative risk, $1.82 ; 95 \%$ CI, 1.194-2.784; $P=.0054)$. Moreover, erlotinib was better tolerated than 4 cycles of adjuvant chemotherapy. Using a similar study design, the North American phase II SELECT compared adjuvant erlotinib (2 years) with historic controls and reported a 2 -year DFS of $86 \%{ }^{6}$
Dose reductions occurred in $40 \%$ of the erlotinib group, and $45 \%$ of patients in the entire 100-patient cohort had pathologic stage I disease.

More recently, the randomized, open-label, phase III ADJUVANT/CTONG1104 clinical trial results were reported. ${ }^{7}$ In this study from China, only patients with node-positive stage II or III LUAD were randomized to adjuvant gefitinib ( 2 years) or 4 cycles of cisplatin and vinorelbine. The primary end point was DFS, which was significantly longer with gefitinib (28.7 months; $95 \% \mathrm{CI}$, 24.9-32.5) than with vinorelbine plus cisplatin (18.0 months, 95\% CI, 13.6-22.3; HR, 0.60, 95\% CI, $0.42-0.87 ; P=.0054)$. Serious toxicities occurred less often in the gefitinib group than in the chemotherapy group. The CTONG1104 data were recently updated-at a median follow-up of 80 months, median OS was 75.5 months in the gefitinib group versus 62.8 months in the chemotherapy group (HR, 0.92; $P=.674$ ). ${ }^{8}$ Thus, the ADJUVANT/ CTONG1104 trial, using a first-generation TKI, failed to show an OS advantage for adjuvant TKI therapy, compared with adjuvant cytotoxic chemotherapy, in locally advanced, surgically resected EGFR-mutant LUAD.

\section{ADAURA}

The phase III FLAURA trial demonstrated osimertinib was superior to gefitinib or erlotinib in terms of progression-free survival $^{1}$ and OS, ${ }^{9}$ supporting osimertinib as the standardof-care first-line therapy for untreated EGFR mutationpositive (Ex19del or L858R) advanced NSCLC. Given these observations and the need to improve outcomes in surgically resected LUAD, ADAURA was conceived (https://www. clinicaltrials.gov/ct2/show/NCT02511106). ADAURA is a phase III, double-blind, randomized international trial designed to assess the efficacy and safety of osimertinib, compared with placebo, in patients with completely resected stage IB-IIIA (7th Edition, Cancer Staging Manual of the American Joint Committee on Cancer) EGFR mutationpositive (Ex19del or L858R) LUAD. ${ }^{9}$ The primary end point is DFS in pathologic stage II and IIIA disease. Secondary end points are OS, DFS in all disease stages (including pathologic stage IB), health-related quality of life, and safety. Patients were stratified according to disease stage, EGFR mutational status (Ex19del or L858R), and race (Asian or non-Asian) and were randomly assigned (1:1) to receive either oral osimertinib (80 mg once daily) or placebo. Screening and randomization occurred after patients had undergone surgery and received adjuvant chemotherapy (60\% in both arms). $\mathrm{Pa}$ tients are planned to receive osimertinib or placebo for 3 years or until disease recurrence or fulfillment of a criterion for discontinuation. Postoperative radiation therapy was not allowed in the study. ${ }^{10}$

The trial began in 2015, and after randomization of 682 patients, enrollment closed in 2019. Following a planned 
review by the independent data monitoring committee in April 2020, the committee recommended the trial be unblinded at the trial level 2 years early because of evidence of an efficacy benefit. Of the 470 patients with stage IIIIIA disease, 156 (33\% maturity) experienced disease recurrence or died. Median follow-up for DFS was 22 months in the osimertinib group versus 15 months in the placebo group. The percentage of patients who were alive and disease-free at 24 months was $90 \%$ in the osimertinib group versus $44 \%$ in the placebo group (overall HR for disease recurrence or death, $0.17,99.06 \%$ CI, 0.11-0.26; $P<.001$; Figure 1 ). This equates to an $83 \%$ reduction in the risk of disease recurrence or death, indicating a significantly longer DFS among patients in the osimertinib group. Although a secondary end point, DFS was better in patients with stage IB disease who received osimertinib: 2-year DFS was $88 \%$ in the osimertinib group versus $71 \%$ in the placebo group (HR, 0.39; 95\% CI, 0.18-0.76).

While the ADAURA data will continue to mature, these dramatic improvements in DFS are contrasted with the DFS results from the LACE (Lung Adjuvant Cisplatin Evaluation) analysis trial, which favored adjuvant chemotherapy (HR, 0.84), with absolute benefits of $5.8 \%$ at both 3 and 5 years. While one may postulate that the benefits of adjuvant osimertinib would impact only rates of distant recurrence, there was also a notable decrease in rates of locoregional recurrence. Locoregional-only recurrence was observed in 23 of 339 patients $(7 \%)$ in the osimertinib group versus 61 of 343 patients $(18 \%)$ in the placebo group. Finally, a major clinical problem in EGFR-mutant LUAD is the development of brain metastases. In ADAURA, CNS recurrence was found in 45 patients (osimertinib, 6; placebo, 39) at a median follow-up of 22 months. The conditional probability of CNS recurrence at 12 months was $<1 \%$ with osimertinib versus $7 \%$ with placebo. Thus, to date, there is an $82 \%$ reduction in the risk of CNS disease recurrence with osimertinib after complete resection of $E G F R$-mutant LUAD. OS data were not reported, secondary to limited follow-up.

The median duration of treatment was 22 months (range, 0-38) in the osimertinib group versus 19 months (range, 0-36) in the placebo group. Adjuvant osimertinib was well tolerated in general, with adverse events of grade $\geq 3$ observed in 68 patients $(20 \%)$ in the osimertinib group and 46 patients $(13 \%)$ in the placebo group. The most common toxicities were diarrhea, paronychia, and dry skin. Importantly, suspected treatment-related interstitial lung disease was mild to moderate in severity, with no deaths. Dose interruptions, dose reductions, and discontinuation of osimertinib owing to adverse events occurred in $80(24 \%), 29(9 \%)$, and $37(11 \%)$ patients, respectively.

The published surgical-specific results of the study are limited. It is important to note that all patients had a lobectomy $(94 \%-97 \%)$ or greater resection-no patient had a sublobar resection, including segmentectomy. We have no

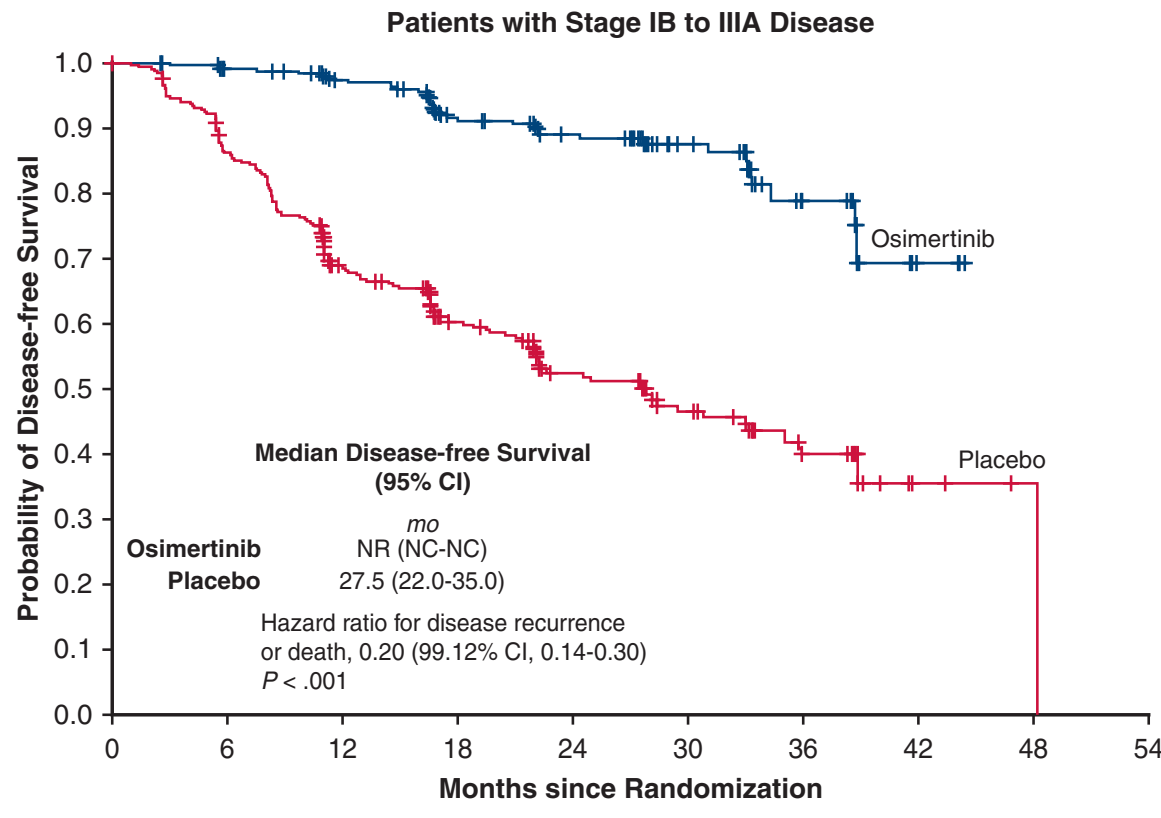

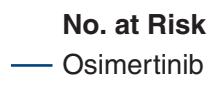

Osimertin
— Placebo
339

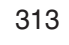

287
272

207

208

148

138

88

74

53
27

20

5

31

FIGURE 1. Kaplan-Meier estimates of the duration of disease-free survival in the overall population of patients with stage IB to IIIA disease. Tick marks indicate censored data. $C I$, Confidence interval; $N R$, not reached; $N C$, could not be calculated. From Wu and colleagues. ${ }^{10}$ 
data on surgical approach (minimally invasive or open) or the quality of the intraoperative nodal dissections performed, and contemporary classification of adenocarcinoma histologic subtypes is limited. On the basis of the results of ADAURA, the Food and Drug Administration approved osimertinib as adjuvant therapy for $E G F R$-mutant (Ex19 Del or L858R) lung cancer in December 2020.

\section{REMAINING ISSUES}

While the use of adjuvant osimertinib in patients with completely resected EGFR-mutant LUAD is paradigmchanging, several unanswered questions remain. First, although the improvement in DFS with osimertinib is profound, will these DFS benefits translate to an improvement in OS? In ADJUVANT/CTONG1104, this was not the case. Other studies, such as OPTIMAL in EGFR-mutant LUAD ${ }^{11}$ and trials in solid tumors, ${ }^{12}$ have failed to see improvements in DFS translate to similar benefits in OS. However, there are several reasons to suspect the benefit may translate in the ADAURA trial. To begin, the magnitude of improvement in DFS is very significant, and given that most patients with disease recurrence die of disease, there is optimism that OS will also be improved with osimertinib, compared with placebo, although likely not at the magnitude of the increase in DFS reported to date. In addition, an improvement in OS was observed in the clinical trials of osimertinib in the metastatic disease setting, suggesting that this may also occur in the adjuvant setting. Finally, in earlier-stage disease, where there are fewer tumor cells and less tumor heterogeneity, the potential for cure with an EGFR inhibitor may be greater. Notwithstanding these observations, we do not yet know the impact of adjuvant osimertinib on OS in this population.

Second, do patients also need adjuvant cytotoxic chemotherapy, or would osimertinib alone suffice? As surgeons, we frequently hear from our patients about the lingering, and sometimes debilitating, longer-term side effects of chemotherapy-particularly the neuropathy. In ADAURA, the decision to offer adjuvant chemotherapy was left to the discretion of the physician. Sixty percent of both cohorts received adjuvant chemotherapy (delivered first and in sequential fashion), administered to approximately $75 \%$ and $25 \%$ of patients with pathologic stage II/IIIA and IB disease, respectively. There was no observed difference in DFS if the patient received adjuvant chemotherapy or not, although these results are immature, and longer follow-up is required. At present, it is expected that clinical guidelines will continue category I recommendations for adjuvant platinum-based chemotherapy, even in EGFR-mutant LUAD, until more-rigorous data suggest adjuvant TKI therapy alone is noninferior.

Third, 3 years of adjuvant osimertinib therapy seems quite long and expensive. In the trial, the median duration of osimertinib therapy was 22 months, and DFS was excellent for all disease stages. How much more does an additional year (or 2) of adjuvant osimertinib improve these alreadyoutstanding outcomes? Similar to the question regarding OS, we will need to wait to see, as only $10 \%$ to $12 \%$ of the entire cohort has received 3 years of treatment. Although the side effects of osimertinib are low-grade, diarrhea, paronychia, and stomatitis did occur in $46 \%, 25 \%$, and $18 \%$ of patients, respectively. Such adverse effects, even if lowgrade, can be challenging when a therapy is given over several years. ${ }^{12}$ However, 3 years of adjuvant osimertinib may be a moot topic, as patients may elect to discontinue the drug and follow-up visits after 1-2 years. Alternatively, advances in the detection of minimal residual disease with ctDNA technologies may help more accurately guide our driver-directed targeted-therapy adjuvant-therapy algorithms and avoid the possibility of overtreatment, which remains a relevant consideration in all adjuvant-therapy trials. ${ }^{13}$

\section{BEYOND ADAURA}

ADAURA opened several exciting possibilities for thoracic surgeons and their patients. While adjuvanttherapy algorithms could be perceived by thoracic surgeons to be the "responsibility" of our medical oncology colleagues, that is incorrect. We must ensure that our patients have reflex testing of their resected NSCLC for actionable EGFR alterations, as well as other relevant genomic perturbations. Smeltzer and colleagues ${ }^{14}$ recently showed that EGFR mutational assessment occurs very commonly worldwide, but there is no specific data on early-stage, surgically resected disease. Additional opportunities related to the identification and targeting of selected driver mutations to improve the care of our patients are rapidly unfolding. More adjuvant TKI trials that exploit other targetable driver mutations are currently being explored. Results will be slower to mature, given the relatively low frequency of these genomic alterations in LUAD, but the trials will be performed. It may be suspected that the primary reason thoracic surgeons have not focused on tumor genomic profiling is that, heretofore, no data have existed to support adjuvant targeted therapies for $\mathrm{R} 0$ resected LUAD. ADAURA has changed that, and the contemporary thoracic surgical oncologist needs to increase their understanding of these emerging therapies.

Similar to the adjuvant osimertinib paradigm is the rapid transition of targeted therapies, which have demonstrated efficacy in advanced-stage disease, to the neoadjuvant setting in patients with surgically resectable disease. Leading this effort is the NeoADAURA trial, an international randomized (1:1:1) phase III trial examining neoadjuvant chemotherapy alone, chemotherapy plus osimertinib, or osimertinib alone for 9 weeks followed by surgical resection for patients with clinical stage II and IIIB disease and $E G F R$-mutant LUAD. The primary outcome is major pathologic response; secondary outcomes are pathologic 
complete response, DFS, and OS. Additional targetedtherapy neoadjuvant trials are soon to open as part of a joint multi-institutional clinical trial effort from the Lung Cancer Mutation Consortium, Lung Cancer Research Foundation, and the American Association for Thoracic Surgery Thoracic Surgery Oncology Group. Using an umbrella trial design, this study has the goal to screen for 10 actionable driver mutations in 1000 patients with lung cancer who are candidates for neoadjuvant therapy. Subsequently, the efficacy and safety of driver-specific neoadjuvant targeted therapies will be examined in a series of industrysupported clinical trials.

\section{CONCLUSIONS}

Tumor genomic profiling in early-stage LUAD has heretofore been primarily associated with prognosis and, more recently, prediction of disease recurrence. ${ }^{15}$ ADAURA is the first "positive" adjuvant clinical trial in surgically resected lung cancer since the trials of adjuvant cytotoxic doublet chemotherapy were reported more than 15 years ago. The questions of impact on OS, duration of therapy, and the role of current guideline-concordant chemotherapy remain relevant, and answers will come with maturation of the ADAURA data. Finally, we can expect more clinical trials in earlier-stage disease-adjuvant and neoadjuvantand these will be based on tumor-specific oncogenic driver genomic perturbations identified through reflex testing.

\section{Conflict of Interest Statement}

Dr Jones serves as a consultant for AstraZeneca and on a Clinical Trial Steering Committee for Merck. Dr Wu serves on the advisory board of AstraZeneca and Boehringer Ingelheim; has received honorarium or speaker fees from AstraZeneca, Roche, Boehringer Ingelheim, Pfizer, BristolMeyers Squibb, Meso Scale Diagnostics, and Eli Lilly; and has received contracted or supported research grants from AstraZeneca, Roche, and Boehringer Ingelheim. Dr Tsuboi reports receiving lecture fees from Johnson \& Johnson Japan, Teijin Pharma, Taiho Pharma, and Medtronic Japan; grant support, paid to his institution, lecture fees, and advisory board fees from AstraZeneca and Merck Sharp \& Dohme; grant support, paid to his institution, and lecture fees from Eli Lilly Japan, Bristol-Myers Squibb, and Ono Pharmaceutical; lecture fees and advisory board fees from Chugai Pharmaceutical; grant support, paid to his institution, from Boehringer Ingelheim Japan, and advisory board fees from Novartis. Dr Herbst reports personal fees from Abbvie Pharmaceuticals, ARMO Biosciences, Biodesix, Bristol-Myers Squibb, EMD Serrano, Genentech/Roche, Genmab, Heat Biologics, Halozyme, Loxo Oncology, Nektar, Novartis, Pfizer, Sanofi, Seattle Genetics, Shire PLC, Spectrum Pharmaceuticals, Symphogen, Tocagen, and
Tesaro, outside the submitted work; grants and personal fees from AstraZeneca, Eli Lilly and Company, and Merck and Company; and personal fees and other from Infinity Pharmaceuticals, Junshi Pharmaceuticals, Neon Therapeutics, and NextCure.

The Journal policy requires editors and reviewers to disclose conflicts of interest and to decline handling or reviewing manuscripts for which they may have a conflict of interest. The editors and reviewers of this article have no conflicts of interest.

\section{References}

1. Soria J-C, Ohe Y, Vansteenkiste J, Reungwetwattana H, Chewaskulyong B, Lee $\mathrm{KH}$, et al. Osimertinib in untreated EGFR-mutated advanced non-smallcell lung cancer. N Engl J Med. 2018;378:113-25.

2. Brandt W, Bouadallah I, Tan KS, Park BJ, Adusumilli PS, Molena D, et al. Predictors of distant recurrence following R0 lobectomy for pN0 lung adenocarcinoma. J Thorac Cardiovasc Surg. 2018;155:1212-24.

3. Pignon JP, Tribodet H, Scagliotti GV, Douillard JY, Shepherd FA, Stephens RJ, et al. Lung adjuvant cisplatin evaluation: a pooled analysis by the LACE collaborative group. J Clin Oncol. 2008;26:3552-9.

4. Kelly K, Altorki NK, Eberhardt WEE, O'Brien MER, Spigel DR, Crinò L, et al. Adjuvant erlotinib versus placebo in patients with stage IB-IIIA non-small-cell lung cancer (RADIANT): a randomized, double-blind, phase III trial. J Clin Oncol. 2015;33:4007-14.

5. Yue D, Xu S, Wang Q, Li X, Shen Y, Zhao H, et al. Erlotinib versus vinorelbine plus cisplatin as adjuvant therapy in Chinese patients with stage IIIA EGFR mutation-positive non-small-cell lung cancer (EVAN): a randomised, openlabel, phase 2 trial. Lancet Respir Med. 2018;11:863-73.

6. Pennell NA, Neal JW, Chaft JE, Azzoli CG, Janne PA, Govindan R, et al. SELECT: a phase II trial of adjuvant erlotinib in patients with resected epidermal growth factor receptor-mutant non-small-cell lung cancer. J Clin Oncol. 2018; 37:97-104.

7. Zhong WZ, Wang Q, Mao W-M, Xu ST, Wu L, Shen Y, et al. Gefitinib versus vinorelbine plus cisplatin as adjuvant treatment for stage II-IIIA (N1-N2) EGFR-mutant NSCLC (ADJUVANT/CTONG1104): a randomised, open-label, phase 3 study. Lancet Oncol. 2018;19:139-48.

8. Zhong WZ, Wang Q, Mao W-M, Xu ST, Wu L, Wei Y-C, et al. Gefitinib versus vinorelbine plus cisplatin as adjuvant treatment for stage II-IIIA (N1-N2) EGFRmutant NSCLC: final overall survival analysis of CTONG1104 phase 3 trial. J Clin Oncol. 2021;39:713-22.

9. Ramalingam SS, Vansteenkiste J, Planchard D, Cho YC, Gray JE, Ohe Y, et al Overall survival with osimertinib in untreated, EGFR-mutated advanced NSCLC. N Engl J Med. 2020;382:41-50.

10. Wu YL, Tsuboi M, He J, John T, Grohe C, Majem M, et al. Osimertinib in resected EGFR-mutated non-small-cell lung cancer. N Engl J Med. 2020;383:1711-23.

11. Zhou C, Wu YL, Chen G, Feng J, Liu XQ, Wang C, et al. Final overall survival results from a randomised, phase III study of erlotinib versus chemotherapy as first-line treatment of EGFR mutation-positive advanced non-small-cell lung cancer (OPTIMAL, CTONG-0802). Ann Oncol. 2015;26:1877-83.

12. Gyawali B, West H. Lessons from ADAURA on adjuvant cancer drug trials: evidence, ethics, and economics. J Clin Oncol. 2021;39:175-8.

13. Isbell JM, Jones DR, Li BT. Circulating tumor DNA: a promising biomarker to guide postoperative treatment and surveillance of non-small cell lung cancer. $J$ Thorac Cardiovasc Surg. 2018;155:2628-31.

14. Smeltzer MP, Wynes MW, Lantuejoul S, Soo R, Ramalingam SS, VarellaGarcia M, et al. The international association for the study of lung cancer global survey on molecular testing in lung cancer. J Thorac Oncol. 2020;15:1434-48.

15. Jones GD, Brandt WS, Shen R, Sanchez-Vega F, Tan KS, Martin A, et al. Prediction of early recurrence using tumor genomic profiling in completely resected early-stage lung adenocarcinoma. JAMA Surg. 2021;156:e205601.

Key Words: lung adenocarcinoma, EGFR, osimertinib, ADAURA 